$\mathbb{P}$ periodica polytechnica

Civil Engineering

54/1 (2010) 3 9

doi: 10.3311/pp.ci.2010-1.01

web: http://www.pp.bme.hu/ci

(c) Periodica Polytechnica 2010

RESEARCH ARTICLE

\section{Distributed traffic control system based on model predictive control}

\author{
Tamás Tettamanti / István Varga
}

Received 2009-06-09, accepted 2009-10-20

\begin{abstract}
The paper investigates a distributed control system scheme for urban road traffic management. The control algorithm is based on model predictive control (MPC) involving Jacobi iteration algorithm to solve constrained and nonlinear programming problem.
\end{abstract}

The signal controllers of traffic network constitute a network of computers. They can distribute their computation realizing an efficient traffic control without any central management. However the optimal control inputs can be also calculated by a single traffic controller if the traffic network contains few intersections.

The control aim is to relieve traffic congestion, reduce travel time and improve homogenous traffic flow in urban traffic area using distributed control architecture. The MPC based control strategy can be implemented in any urban transportation network but adequate measurement system and modern traffic controllers are needed. Theory, realization possibilities and simulation of the control method are also presented. The simulation results show that the system is able to ameliorate the network efficiency and reduce travel time. The distributed MPC based traffic control strategy proves the effectiveness by realizing a dependable control operation and creating optimal flow in the network subjected to control input constraints.

\section{Keywords}

urban traffic control $\cdot$ model predictive control $\cdot$ distributed traffic control system $\cdot$ Jacobi algorithm

\section{Tamás Tettamanti}

Department of Control and Transport Automation, BUTE, Bertalan L. u. 2, Budapest, H-1111, Hungary

e-mail: tettamanti@mail.bme.hu

\section{István Varga}

Systems and Control Laboratory, Computer and Automation Research Institute, Hungarian Academy of Sciences, Kende u. 13-17., H-1111 Budapest, Hungary e-mail: ivarga@sztaki.hu

\section{Introduction}

More and more people are concerned about the negative phenomenon resulted by the negative effects of the growing traffic motorization. Traffic congestion is the primary direct impact which became everyday occurrence in the last decade. As world trade is continuously increasing, it is obvious that congestions represent also a growing problem. The capacity of the traffic networks saturates during rush hours. At the same time, the traditional traffic management is getting less effective in sustaining a manageable traffic flow. As a result, external impacts appear causing new costs for the societies.

The development of new control strategies is a real demand of nowadays. In case the distance is relatively short between several intersections with traffic lights, it is advisable to coordinate the operation of the intersection controller devices.

In case of designing a traffic control system one has to face two important problems. Naturally, an efficient and dependable control algorithm is a primary object. However designing suitable control system architecture is also indispensable, since a control algorithm has several operation requirements.

One of the possible solutions for an efficient control algorithm is the practical application of modern control theory. In case of designing urban traffic control using state space theory, it is advisable to choose a simple, possibly linear model. A model like this is the store-and-forward approach [8] which describes the queue building before the stop line. This model is also the base of Papageorgiou's urban control strategy in the TUC model [5]. The TUC system implements an LQ control algorithm which is not able to satisfy the constraints of the system. A possible solution can be the use of MPC [11] which is able to take these constraints into consideration.

The classical scheme for adaptive road traffic management structure is based on control center which processes and computes all signal control for the network. Another method for the control system architecture is the decentralized and distributed control scheme. This approach has numerous economical and technological advantages.

In the paper we present a distributed control system scheme for urban road traffic management which operation is based on 


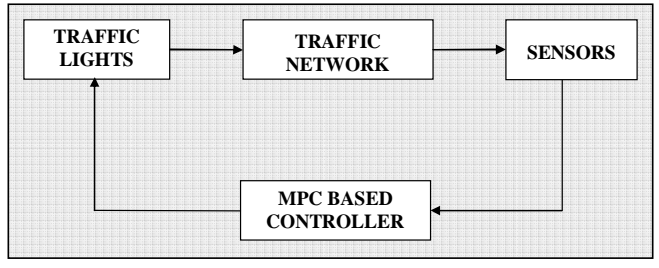

Fig. 1. The control loop of the traffic control system

MPC. The control aim is to relieve congestion, reduce travel time and maintain homogenous traffic flow in urban traffic network.

To test and validate our control strategy and architecture design we created a simulation program. The simulation results show that the system is able to ameliorate the network efficiency and reduce travel time. The MPC based traffic control strategy proves effectiveness by creating optimal flow in the network subjected to control input constraints.

\section{Distributed control system scheme in urban road traffic network}

The traffic control problem can be identified as a traditional control problem with a plant and feedback control as shown in Subsec. 3. The actuators are the traffic lights at the junctions. Sensors are loop detectors or cameras.

Generally, the architectures of traffic control systems can be central, distributed (decentralized), or mixed. The central management architecture is a frequent strategy based on a central processor which controls all signal controllers in the transportation network. Distributed and mixed control systems are not so common applications yet. However they have many advantages and represent a new way in traffic control technology. Decentralized management systems carry a higher performance since they can distribute their computations between the traffic controllers. As well as they represent a higher operation safety because of their structural redundancy. Some of these distributed realizations are for example SCATS [19] or Utopia [17].

The aim of our research is to elaborate a control process for decentralized management architecture. The system has to perform the control of all the traffic lights in its sphere of action in a coordinated way depending on the actual state of traffic. The controller must be able to dynamically create the traffic signal set of the intersections. From the point of view of realization, this means that before every period a new traffic sign must be generated regarding all the traffic lights, in harmony with the actual traffic. To solve these requirements, one must choose a method which is able to take all the constraints into consideration in course of the control input setting and can be implemented in a distributed control system scheme. Regarding the above, the MPC based technology is suitable to fulfil these demands [7, 11, 12]. The goal of this algorithm is translated into a cost function expressed in terms of some traffic states (i.e. traffic flows, traffic densities, average speeds). As the name cost func-

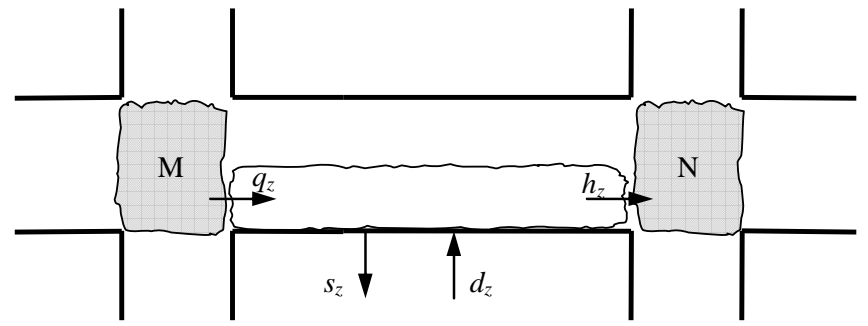

Fig. 2. Store-and-forward model

tion states, the cost is associated with an undesirable traffic state with congestion which needs to be minimized. Accordingly the system intends to find the lowest value of the cost function.

The MPC strategy is a state-of-the-art technology which can be implemented in any urban road transportation network. On the other hand there are some preliminary conditions the network has to correspond to. Adequate measurements system and traffic controllers are indispensable for the functioning of the optimization process.

\section{Traffic modeling using state space theory}

To create an efficient control design an adequate traffic model is needed. Transportation model has to describe the accurate dynamics of the traffic processes within the controlled traffic network. Using traffic models it is possible to simulate traffic processes faster than real-time. This allows a real-time evaluation and optimization of different alternative control strategies that would not be possible otherwise.

A wide variety of traffic models exists. These models can be classified based on their properties [3]. The state space traffic models describe traffic flow in combination with advanced (adaptive) control design technologies. Hence a state space traffic model approach is used in our control system.

The chosen traffic flow model contains the so called storeand-forward model (Fig. 2] [8] which is similar to the model used in TUC [5,6] urban traffic control strategy. Fig. 2 shows the coherence of two neighboring intersections $(M, N)$ in the transportation network where $q_{z}$ is the inflow, $h_{z}$ is the outflow, $d_{z}$ and $s_{z}$ are the demand and the exit flow of link $z$.

In the case of traffic networks with multiple ramifications, for the portrayal of vehicles movement in the space state, one proposed the following discrete time stochastic LTI state equation:

$$
\begin{gathered}
x_{z}(k+1)=x_{z}(k)+ \\
T\left[\left(1-\kappa_{z, 0}\right) \sum_{w \in I_{M}} \alpha_{w, z} \frac{S_{w} \sum_{i \in v_{w}} g_{M, i}(k)}{C}-\frac{S_{z} \sum_{i \in v_{z}} g_{N, i}(k)}{C}\right],
\end{gathered}
$$

where $x(k)$ is the state vector, representing the number of vehicles standing in a certain branch of the intersection, green time $g(k)$ is the control input. $\alpha_{w, z}$ are the entering turning rates which are considered constants. The values of turning rates have strong influence on the system's operation. The accurate information of them represents an important problem in control de- 
sign. Turning rates are not easily measurable parameters. One of the good solutions can be the estimation [9]. $\kappa$ represents the fixed and known exit rates. $S$ denotes saturation flow, $C$ means the cycle time, $T$ is the control interval, and $k=1,2 \cdots$ a is the discrete time index. Index $j$ denotes the junction identifier, index $i$ means the stage. The cycle time, the lost times of each junction are fixed and it means that the sum of the green times is also fixed in each junction.

The state and the measurement equation can be written in general form as:

$$
\begin{aligned}
& x(k+1)=A x(k)+B g(k)+x_{i n}(k)+w(k) \\
& y(k)=C x(k)+v(k),
\end{aligned}
$$

where $x_{i n}$ means the number of input vehicles, $w$ is the sum of the non-measurable fault/noise, while $v$ is the measurement noise. The demands to enter at the boundaries of the network are considered as measurable fault/noise.

\section{Optimal solution of the MPC cost function using Ja- cobi algorithm}

To solve an MPC problem means the minimization of an adequate cost function which contains the states of the controlled system. Thus, the first step to realize our control is designing the state space representation of the traffic network. The state equation is created using measurable traffic parameters. The cost function contains the matrixes of this state space equation.

The final goal of this algorithm is to minimize the queue lengths at the stop lines which yields to the maximization of the number of vehicles passing the given transportation network.

\subsection{MPC cost function}

The state space equation for MPC design can be given as follows:

$$
\begin{aligned}
\underbrace{\left[\begin{array}{c}
x(k+1 \mid 1) \\
x(k+1 \mid 2) \\
\vdots \\
x(k+1 \mid N)
\end{array}\right]}_{x(k+1)} & =\underbrace{\left[\begin{array}{c}
x(k)+x_{i n}(k) \\
x(k)+2 x_{i n}(k) \\
\vdots \\
x(k)+N x_{i n}(k)
\end{array}\right]}_{c(k)} \\
+ & \underbrace{\left[\begin{array}{cccc}
T & 0 & \ldots & 0 \\
T & T & \ldots & 0 \\
\vdots & \vdots & \ddots & \vdots \\
T & T & \ldots & T
\end{array}\right]}_{B} \underbrace{\left[\begin{array}{c}
g_{g(k \mid 0)} \\
g(k \mid 1) \\
\vdots \\
g(k \mid N-1)
\end{array}\right]}_{g(k)}
\end{aligned}
$$

where $x$ is a hyper vector of the state vectors, representing the number of vehicles standing at each branch of the intersections, $c$ is a hyper vector of the combination of the previous state vectors and the number of input vehicles vectors $\left(x_{i n}\right)$. Automobiles entering the controlled traffic network are considered input vehicles. $B$ is a lower triangular hyper matrix including the matrix of turning rates of the traffic network $(T), g$ is hyper vector of the control input vectors (green times), $k=1,2 \ldots$ a is the discrete time index, and $N$ is the length of the MPC horizon.

The MPC cost function is characterized by the weighted system states and control inputs (5).

$$
J(k)=\frac{1}{2}\left\{q x^{T}(k) x(k)+r g^{T}(k) g(k)\right\} \rightarrow \min
$$

The minimization of the cost function leads to the minimization of the vehicle queues waiting for crossing intersections. The control input green time is defined corresponding to the states of intersection branches representing a fully adaptive traffic management. Where $q=0.1$ and $r=1$ are appropriately chosen tuning parameters. The weightings reflect that the control input variation is lightly punished compared to the state variation. The selection of the appropriate weightings is important, because this could influence (especially the end-point weight) the stability of the closed loop [10].

Different stability proofs exist for receding horizon control algorithms. However, [11,13] offer different methodological approaches, one prefers using a predefined terminal set (based on the solution of the Algebraic Riccati Equation of a steady state LQ feedback problem) [13,20]. The terminal set is subjected to the control input's constraints. Therefore, the solution of the finite horizon minimization can be interpreted as an optimal state feedback driving the closed loop into an invariant set. Substituting $x(k)$ and $g(k)$ in (5) one arrives to:

$$
\begin{aligned}
J(k) & =\frac{1}{2} g^{T}\left(q B^{T} B+r I\right) g+q c^{T} B g+\frac{1}{2} q c^{T} c \\
& =\frac{1}{2} g^{T} \Phi g+\beta^{T} g+\gamma
\end{aligned}
$$

As $\gamma$ is a constant term, finally one has the objective function to minimize:

$$
J(k)=\frac{1}{2} g^{T}(k) \Phi g(k)+\beta^{T}(k) g(k) \rightarrow \min
$$

where

$$
\Phi=\left[\begin{array}{ccc}
\varphi_{11} & \ldots & \varphi_{1 n} \\
\vdots & \ddots & \vdots \\
\varphi_{n 1} & \ldots & \varphi_{n n}
\end{array}\right], \quad \beta=\left[\begin{array}{c}
\beta_{1} \\
\vdots \\
\beta_{n}
\end{array}\right]
$$

$\Phi$ is constant matrix as it contains the combination of constant turning rates and fixed tuning parameters. At the same time, $\beta$ contains values coming from the geometry of the traffic area.

\subsection{Constraints of the system}

The control objective of TUC is the minimization and balancing of the numbers of vehicles within the streets of the controlled network. This control objective is approached through the appropriate manipulation of the green splits at urban signalized junctions, assuming given cycle times and offsets. The TUC has some alternative control laws but the main concept is based on LQ and LQI control theory. 
However, a control solution had been searched for which was able to satisfy the two types of constraints on the control input: the positive green times criterion and the linear combinations of green times in each intersection coming from the geometry of the intersections.

$$
\begin{array}{cl}
g_{i} & \geq 0 \\
\sum_{i=1}^{O_{j}} g_{i} & \leq t_{j}^{\mathrm{MAX}} \quad \forall i
\end{array}
$$

where $O_{j}$ is the number of vehicles' columns in intersection $j, J$ is the number of controlled intersections. The constraints can be also expressed as a matrix inequality:

$$
A g \leq b
$$

where matrix $A$ and vector $b$ incorporate all constraints of the system.

By employing the predictive control model, the dynamic determination (per cycle) of the traffic light's period is possible even with the consideration of the natural constraints existing in the system. A method had been elaborated for designing an MPC controller which minimizes the number of vehicles in queue. The controller ensures the control input in order to minimize the functional (7) whereas satisfying dynamic equation (4), measurement equations (5), (6) and constraints (9), (10).

\subsection{Multivariable nonlinear programming}

The solution of the MPC cost function (7) represents a multivariable nonlinear problem subject to linear constraints (11). It formulates a standard quadratic optimization problem [4]:

$$
\begin{aligned}
& J(k)=\frac{1}{2} g^{T} \Phi g+\beta^{T} g \rightarrow \min \\
& \text { s. t. } \quad A g-b \leq 0 .
\end{aligned}
$$

If $\Phi$ is a positive semi definite matrix, (12) gives a convex optimization problem [2]. Otherwise one has to use the singular value decomposition method to $\Phi$ which results a convex problem. This means a linear transformation to the original problem (12).

Using the duality theory [4] the primal problem can be formulated into Lagrange dual standard form. The basic idea in Lagrangian duality is to take the constraints into account by augmenting the objective function with a weighted sum of the constraint functions. We define the Lagrangian associated with the problem as:

$$
L(g, \lambda)=J(k)+\lambda^{T}(A g-b)
$$

We refer to $\lambda_{i}$ as the Lagrange multiplier associated with the $i$ th inequality constraint of (12). The dual function is defined as the minimum value of the Lagrangian function. This can be easily calculated by setting gradient of Lagrangian to zero [2]. This yields an optimal green time vector 17 which minimizes the primal problem. Hence one arrives to the dual of the quadratic programming problem:

$$
\begin{aligned}
& J_{\text {DUAL }}(k)=\frac{1}{2} \lambda^{T} P^{T} \lambda+w^{T} \lambda \rightarrow \min \\
& \text { s. t. } \quad \lambda \geq 0,
\end{aligned}
$$

where $P$ and $w$ are coming from the original problem:

$$
\begin{aligned}
& P=A \Phi^{-1} A^{T}, \\
& w=A \Phi^{-1} \beta+b
\end{aligned}
$$

It is shown that if $\lambda^{*}$ provides optimal solution for the $J_{D U A L}(k)$ problem then

$$
g^{*}=-\Phi^{-1}\left(\beta+A^{T} \lambda^{*}\right)
$$

gives also an optimal solution for the primal problem [15].

The dual problem has a simple constraint set compared with the primal problem's constraints. Hence (14) represents a standard minimization problem over nonnegative orthant which can be resolved by several numerical algorithms.

A very efficient method, the Jacobi iteration was found to solve the optimization problem. Since $\Phi$ is a positive semi definite matrix the $j$ th diagonal element of $P$, given by

$$
p_{j j}=a_{j}^{T} \Phi^{-1} a_{j}
$$

is positive. This means that for every $j$ the dual cost function is strictly convex along the $j$ th coordinate. Therefore the strict convexity is satisfied and it is possible to use the nonlinear Jacobi algorithm [4]. Because the dual objective function is also quadratic the iteration can be written explicitly. Taking into account the form of the first partial derivative of the dual cost

$$
w_{j}+\sum_{k=1}^{n} p_{j k} \lambda_{k}
$$

the method is given by:

$$
\begin{gathered}
\lambda_{j}(t+1)=\max \left\{0, \lambda_{j}(t)-\frac{\kappa}{p_{j j}}\left(w_{j}+\sum_{k=1}^{n} p_{j k} \lambda_{k}(t)\right)\right\}, \\
j=1, \ldots, n
\end{gathered}
$$

Where $\kappa>0$ is the stepsize parameter which should be chosen sufficiently small and some experimentation may be needed to obtain the appropriate range for $\kappa$. Convergence can be shown when $\kappa=n^{-1}$ but this value may lead to an unnecessarily slow rate of convergence for some problems.

The importance of this method, over its efficiency, is the ability to satisfy the positivity since equation 20 excludes negative solution for $\lambda$. During the MPC control process at each $(k$ th) step the optimal green times can be directly calculated from equation (17) after solving the problem (12). 


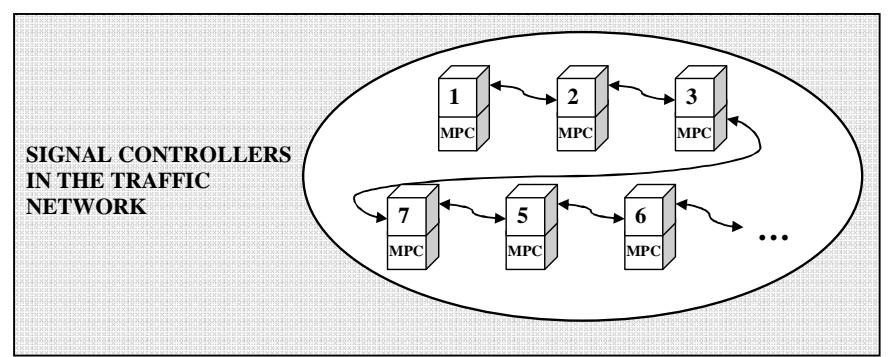

Fig. 3. Decentralized control system scheme

\section{Realization of the MPC based distributed traffic con- trol system}

The economical and technological innovation of the above described control method is represented by the state-of-the-art control design and the optional decentralized realization at the same time.

The technology can be used in any road traffic network which is equipped by adequate signal controllers and detectors, as well as communication between controllers is also required. However no central management is needed to operate and this produces many advantages compared to the classical centralized traffic control system. In the distributed control scheme the computation is executed by the signal controllers' CPUs. They communicate with each other at least at the beginning of every operation cycles. The decentralized control system is represented on Fig. 3.

Generally one signal controller device belongs to one intersection. Nevertheless, some state-of-the-art control devices are able to manage more than one intersection. For example ACTROS VTC 3000 [1] may control the signal sets of maximum three junctions at simultaneously. ACTROS was used to test our control algorithm which will be presented later in Sec. 6.2.

The control system scheme is designed in a decentralized way without any central processor. Moreover the computing process can be distributed between the signal controllers. Since the solution of the Jacobi algorithm 20, is an iteration process the computers can distribute their calculations during operation cycle. After some iteration steps the controllers transmit their computational results to the next controllers until the solution is achieved. Hence in case of large traffic network the signal controllers can resolve the calculation in a distributed way. Naturally the computational need must be portioned uniformly.

If one wishes to control small traffic network with couple of intersections the distributed solution is not certainly required. As the calculation of one Jacobi iteration step means simple multiplications and additions of scalars in case of few intersections a single controller's performance is sufficient to compute all signal sets of the network. For example: for a network consisting twelve branches the computational time is less than $1 \mathrm{sec}$ using a modern signal controller device (see Sec. 6.2). In that case the system is working with redundancy which is very useful at the same time. The controllers can continuously check their

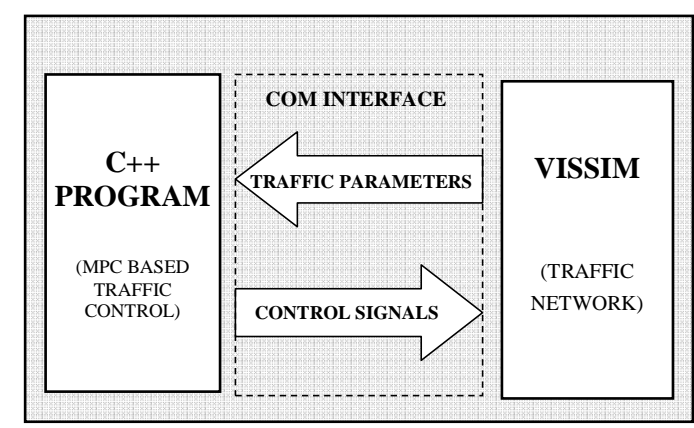

Fig. 4. The simulation environment

operation comparing their computation results. On the other hand, if one of the signal controllers fails in the network the system can go on with safe functioning.

However with the growth of the number of the system states (number of branches of intersections) the computational demand increases quadratically. Thus larger network requires the distributed solution of the MPC control. Certainly the solution method also largely depends on the performance of the actual signal controllers.

\section{Simulation and results}

To test and verify the designed control system scheme one created a simulation environment furthermore used a real signal controller device.

\subsection{Simulation of the MPC based distributed control design}

To verify the designed control system scheme a closed loop simulation environment was created. For traffic network modeling we used VISSIM [18]. This software is a microscopic traffic simulator for analyzing traffic operations. It is able to simulate network consisting of several intersections and allow the use of external control algorithm in the control processes. VISSIM uses a so-called psycho-physical driver behavior model based on the car-following model of Wiedemann. As the model is microscopic it describes all the vehicles moving in the system representing accurate simulation. Access to model data and simulations in VISSIM is provided through a COM interface [14], which allows VISSIM to work as an automation server and to export the objects, methods and properties. Hence COM interface programming was used to create our complex simulation model and control system for VISSIM. The MPC control algorithm was written in Microsoft Visual $\mathrm{C}++$. The created closed loop simulation environment is shown on Fig. 4.

The traffic network used for simulation consists of 4 junctions with twelve controlled branches. The $\mathrm{C}++$ program gets traffic parameters via COM interface from VISSIM. After minimizing the cost function, the new control signals arrive to the traffic simulator on the same path. The process runs every $60 \mathrm{sec}$ while VISSIM is working continuously.

To prove the applicability of the MPC based distributed control design it was compared with classical fixed time control system simulated also in VISSIM. The same input traffic volumes 
Tab. 1. Simulation results of the four junction traffic network

\begin{tabular}{cccc}
\hline Parameter & $\begin{array}{c}\text { Fixed time } \\
\text { control }\end{array}$ & $\begin{array}{c}\text { MPC based } \\
\text { distributed control }\end{array}$ & Change \\
\hline Total travel time per vehicle [sec] & 110 & 96 & $\downarrow 13 \%$ \\
\hline Average speed [km/h] & 18.4 & 23.6 & $\uparrow 28 \%$ \\
\hline Average delay time per vehicle [sec] & 71 & 52 & $\downarrow 27 \%$ \\
\hline Average number of stops per vehicles & 1.5 & 1.2 & $\downarrow 20 \%$ \\
\hline
\end{tabular}

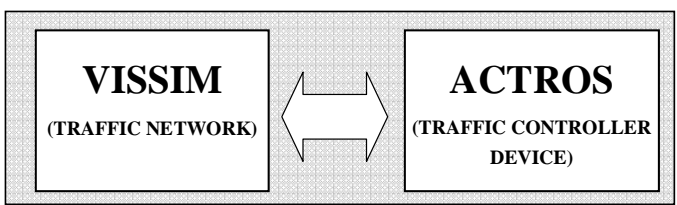

Fig. 5. The VISSIM-ACTROS system

were set for both simulations. The simulation results show that the MPC based distributed system could manage the traffic more efficiently compared with the classical control system. The results are presented in Tab. 1. All important traffic parameters changed in a right way. The total travel time means an average time which is needed to cross the test network. This value reduced by more than $10 \%$. Average speed value augmented the most intensively which is due to the diminution of the stops before the traffic lights. Average delay time gives the difference between the ideal (network without any traffic and traffic lights) and the necessary travel time to cross the traffic area. As a consequence of the previous results the average delay time also reduced strongly. The simulation time was 2 hour.

The aim of the MPC based control is the minimization of the number of vehicles waiting at the stop line. The classical control system cannot adapt to increased volume. The average queue length grew strongly during the simulations. However, the MPC strategy is able to manage heavier traffic situations real-time.

It shows unambiguously the effectiveness of the MPC based system as controller. On the other hand the signal controllers provide the same solutions in distributed as in central architecture [16]. This represents an important advantage since the local signal controllers can solve easily the control problem (see Sec.4.3. No central processor is needed.

\subsection{Testing on a real intersection signal controller}

To verify if our algorithm can run smoothly on a modern signal controller we used the device ACTROS VTC 3000. ACTROS includes an industrial computer running JAVA applications. Hence a JAVA program was also created to run MPC algorithm. VISSIM was connected to ACTROS via LAN communication (Fig. 5).

As expected the controller could operate without any problem. It could suitably manage the previously used traffic network (see Sec. 6.1) in a real time functioning. The computing time of the iteration process is less than $1 \mathrm{sec}$ which is time is not significant. These results show that the required computational demand is very small for a modern signal controller's CPU in case of few controlled intersections.

\section{Conclusion}

Several alternative urban traffic management methodologies and implementation technologies are in use. However the existing methods cannot incorporate physical constraints of the system and/or have operational disadvantages resulted by their control system architectures. To avoid these problems a new, distributed traffic control system scheme was designed.

The control algorithm uses a model based and constrained predictive terminology which is able to take the constraints into consideration. The solution of the problem represents a multivariable nonlinear optimization involving Jacobi iteration. Furthermore, the algorithm can be implemented in a decentralized traffic control system which yields numerous advantages.

The control design was tested using VISSIM traffic simulator and also a real signal controller. The simulation results show that the MPC based control is able to suitably manage the urban traffic control problem and the distributed system operates with significant efficiency and safety. Moreover such control algorithm was found which allows its use in nowadays signal controllers. Generally speaking, the designed strategy can provide effective traffic flow and stable operation in the traffic network.

\section{Acknowledgement}

The authors gratefully acknowledge to the support by the Hungarian National Scientific Research Fund (OTKA CNK78168).

\section{References}

1 ACTROS traffic controller, available at http://www. signalbau-huber. hu/pageVilatiEnglisch/indexEnglish.htm

2 Boyd S, Vandenberghe L, Convex optimization, Cambridge University Press, 2004. ISBN 0521833787.

3 Bellemans T, De Schutter B, De Moor B, Models for traffic control, Delft University of Technology, Fac. of Information Technology and Systems, 2002.

4 Bertsekas DP, Tsitsiklis JN, Parallel and distributed computation: $\mathrm{Nu}$ merical methods, Old Tappan, NJ (USA); Prentice Hall, 1997. ISBN 1886529-01-9.

5 Diakaki C, Dinopoulou V, Aboudolas K, Papageorgiou M, Ben-Shabat E, Seider E, Leibov A, Extensions and new applications of the TrafficResponsive Urban Control Strategy, Transportation Research Record: Jour- 
nal of the Transportation Research Board 1856 (2003), 202-211, DOI 10.3141/1856-22.

6 Diakaki C, Papageorgiou M, McLean T, Integrated traffic-responsive urban control strategy IN-TUC: Application and Evaluation in Glasgow, 1998. DACCORD Workshop on Advanced Motorway Traffic Control.

7 García CE, Prett CE, Morari M, Model predictive control: Theory and practice - A survey, Automatica 25 (1989), DOI 10.1016/00051098(89)90002-2.

8 Gazis DC, Optimal control of oversaturated store-and-forward transportation networks, Transp. Sci. 10 (1976), 1-9, DOI 10.1287/trsc.10.1.1.

9 Kulcsár B, Varga I, Bokor J, Constrained Split Rate Estimation by Moving Horizon, 16th IFAC World Congress, 2005.

10 Kwon HK, Pearson AE, On Feedback Stabilization of Time-Varying Discrete Linear System, IEEE Trans. Autom. Control 23 (1978), 479-481.

11 Maciejowski JM, Predictive Control with Constraints, Harlow, England, Prentice Hall, 2002.

12 Mayne DQ, Rawlings JB, Rao CV, Scokaert PO, Constrained Model Predictive Control: Stability and Optimality, Vol. 36/6, 2000.

13 Rawlings JB, Muske KR, The Stability of Constrained Receding Horizon Control, IEEE Trans. Autom. Control 38 (1993), 1512-1516, DOI $10.1109 / 9.241565$.

14 Roca V, User Manual for the VISSIM COM interface, PTV Planung Transport Verkehr AG, 2005.

15 Rockafellar RT, Convex analysis, Princeton University Press, 1970.

16 Tettamanti T, Varga I, Kulcsár B, Bokor J, Model predictive control in urban traffic network management, 16th Mediterranean Conference on Control and Automation (Ajaccio, Corsica, France, 25 Jun 2008), 2008, pp. 15381543. (CD ISBN:978-1-4244-2505-1, CD:/data/papers/0192.pdf).

17 UTOPIA, available at http://www.peektraffic.nl/page/722

18 Vissim PTV AG, available at wWw.vissim.de

19 Wolshon B, Taylor WC, Analysis of intersection delay under real-time adaptive signal control, Transportation Research Part C: Emerging Technologies 7 (1999), DOI 10.1016/S0968-090X(99)00011-X.

20 Van Der Zijpp NJ, Hamerslag R, Improved Kalman filtering approach for estimating origin-destination matrices for freeway corridors, Transportation Research Record 1443 (1994), 56-64. 